\title{
Multiplicity of Positive Solutions for Weighted Quasilinear Elliptic Equations Involving Critical Hardy-Sobolev Exponents and Concave-Convex Nonlinearities
}

\author{
Tsing-San Hsu and Huei-Li Lin \\ Center for General Education, Chang Gung University, Kwei-Shan, Taiwan \\ Correspondence should be addressed to Tsing-San Hsu, tshsu@mail.cgu.edu.tw \\ Received 24 September 2011; Revised 20 December 2011; Accepted 16 January 2012 \\ Academic Editor: Martin D. Schechter
}

Copyright (C 2012 T.-S. Hsu and H.-L. Lin. This is an open access article distributed under the Creative Commons Attribution License, which permits unrestricted use, distribution, and reproduction in any medium, provided the original work is properly cited.

By variational methods and some analysis techniques, the multiplicity of positive solutions is obtained for a class of weighted quasilinear elliptic equations with critical Hardy-Sobolev exponents and concave-convex nonlinearities.

\section{Introduction and Main Results}

Let $\Omega$ be a smooth bounded domain in $\mathbb{R}^{N}(N \geq 3)$ and $0 \in \Omega$. We will study the multiplicity of positive solutions for the following quasilinear elliptic problem:

$$
\begin{gathered}
-\operatorname{div}\left(|x|^{-a p}|\nabla u|^{p-2} \nabla u\right)-\mu \frac{|u|^{p-2} u}{|x|^{p(a+1)}}=\frac{|u|^{p^{*}(a, b)-2} u}{|x|^{b p^{*}(a, b)}}+\lambda \frac{|u|^{q-2} u}{|x|^{p^{*}(a, d)}} \quad \text { in } \Omega, \\
u=0 \quad \text { on } \partial \Omega,
\end{gathered}
$$

where $\lambda>0,1<p<N, 0 \leq \mu<\bar{\mu}, \bar{\mu} \triangleq((N-p) / p-a)^{p}, 0 \leq a<(N-p) / p, a \leq b, d<a+1$, $1 \leq q<p, p^{*}(a, d) \triangleq N p /(N-p(a+1-d))$ is the critical Sobolev-Hardy exponent. Note that $p^{*}(0,0)=p^{*} \triangleq N p /(N-p)$ is the critical Sobolev exponent. 
In this paper, $W \triangleq W_{a}^{1, p}(\Omega)$ denotes the space obtained as the completion of $C_{0}^{\infty}(\Omega)$ with respect to the norm $\left(\int_{\Omega}|x|^{-a p}|\nabla u|^{p} d x\right)^{1 / p}$. The energy functional of (1.1) is defined on $W$ by

$$
J_{\lambda}(u)=\frac{1}{p} \int_{\Omega}\left(|x|^{-a p}|\nabla u|^{p}-\mu \frac{|u|^{p}}{|x|^{p(a+1)}}\right) d x-\frac{1}{p^{*}(a, b)} \int_{\Omega} \frac{|u|^{p^{*}(a, b)}}{|x|^{b p^{*}(a, b)}} d x-\frac{\lambda}{q} \int_{\Omega} \frac{|u|^{q}}{|x|^{d p^{*}(a, d)}} d x .
$$

Then $J_{\curlywedge} \in C^{1}(W, \mathbb{R}) . u \in W \backslash\{0\}$ is said to be a solution of $(1.1)$ if $\left\langle J_{\lambda}^{\prime}(u), v\right\rangle=0$ for all $v \in W$ and a solution of (1.1) is a critical point of $J_{\lambda}$. By the standard elliptic regularity argument, we deduce that $u \in C^{1}(\Omega \backslash\{0\})$.

Problem (1.1) is related to the following Hardy inequality [1]:

$$
\left(\int_{\mathbb{R}^{N}} \frac{|u|^{p^{*}(a, b)}}{|x|^{b p^{*}(a, b)}} d x\right)^{p / p^{*}(a, b)} \leq C \int_{\mathbb{R}^{N}}|x|^{-a p}|\nabla u|^{p} d x, \quad \forall u \in C_{0}^{\infty}\left(\mathbb{R}^{N}\right)
$$

which is also called the (general or weighted) Hardy-Sobolev inequality. For the sharp constants and extremal functions, see $[2,3]$. If $b=a+1$, then $p^{*}(a, b)=p$ and the following (general or weighted) Hardy inequality holds [1,4]:

$$
\int_{\mathbb{R}^{N}} \frac{|u|^{p}}{|u|^{p(a+1)}} d x \leq \frac{1}{\bar{\mu}} \int_{\mathbb{R}^{N}}|x|^{-a p}|\nabla u|^{p} d x, \quad \forall u \in C_{0}^{\infty}\left(\mathbb{R}^{N}\right),
$$

where $\bar{\mu}=((N-p) / p-a)^{p}$ is the best Hardy constant.

In the space $W$, we employ the following norm if $\mu<\bar{\mu}$ :

$$
\|u\|=\|u\|_{W} \triangleq\left(\int_{\Omega}\left(|x|^{-a p}|\nabla u|^{p}-\mu \frac{|u|^{p}}{|x|^{p(a+1)}}\right) d x\right)^{1 / p}
$$

By (1.4) it is equivalent to the usual norm $\left(\int_{\Omega}|x|^{-a p}|\nabla u|^{p} d x\right)^{1 / p}$ of the space $W$. According to (1.4), we can define the following best constant for $0 \leq a<(N-p) / p, a \leq b<a+1$ and $\mu<\bar{\mu}$ :

$$
S_{\mu, a, b}(\Omega)=\inf _{u \in W \backslash\{0\}} \frac{\|u\|^{p}}{\left(\int_{\Omega}|u|^{p^{*}(a, b)} /|x|^{b p^{*}(a, b)} d x\right)^{p / p^{*}(a, b)}} .
$$

From Kang [5, Lemma 2.2], $S_{\mu, a, b}$ is independent of $\Omega \subset \mathbb{R}^{N}$. Thus, we will simply denote that $S_{\mu, a, b}(\Omega)=S_{\mu, a, b}\left(\mathbb{R}^{N}\right)=S_{\mu, a, b}$. 
When $a=0$, we set $s=d p^{*}(0, d)$ and $t=b p^{*}(0, b)$, then (1.1) is equivalent to the following quasilinear elliptic equations:

$$
\begin{gathered}
-\operatorname{div}\left(|\nabla u|^{p-2} \nabla u\right)-\mu \frac{|u|^{p-2} u}{|x|^{p}}=\frac{|u|^{p^{*}(t)-2} u}{|x|^{t}}+\lambda \frac{|u|^{q-2} u}{|x|^{s}} \text { in } \Omega, \\
u=0 \quad \text { on } \partial \Omega,
\end{gathered}
$$

where $\lambda>0,1<p<N, 0 \leq \mu<\bar{\mu}=((N-p) / p)^{p}, 0 \leq s, t<p, 1 \leq q<p$ and $p^{*}(t)=$ $p(N-t) /(N-p)$.

Such kind of problem relative with (1.7) has been extensively studied by many authors. When $p=2$, people have paid much attention to the existence of solutions for singular elliptic problems (see [6-16] and their references therein), besides, in the most of these papers, the operator $-\Delta-\mu /|x|^{2}$ with Sobolev-Hardy critical exponents (the case that $t=0$ ) has been considered. Some authors also studied the singular problems with SobolevHardy critical exponents (the case that $t \neq 0$ ) (see [17-22] and their references therein). In $[23,24]$, the authors deal with doubly-critical exponents.

When $p \neq 2$. The quasilinear problems related to Hardy inequality and Sobolev-Hardy inequality have been studied by some authors [25-32]. Here we recall the work in [25], where the extremal functions for the best Sobolev constant $S_{\mu, 0,0}$ were studied. The results can be employed to study the problems with critical Sobolev exponents and Hard terms, see [25, 28]. In [26] it is investigated in $\mathbb{R}^{N}$ a quasilinear elliptic equation involving doubly critical exponents by the concentration compactness principle [33, 34].

We should note that the nonlinearities of problems studied in $[11-14,26,28,31]$ are all not sublinear or $p$-sublinear near the origin. To the best of our knowledge, there are few results of problem (1.7) with nonlinearities being $p$-sublinear near the origin for $1<p<N$. We are only aware of the works $[20,30,32]$ which studied the existence and multiplicity of positive solution of problem (1.7) with $1 \leq q<p<N$. In this paper, we study (1.1) and extend the results of $[20,30,32]$ to the case $a \neq 0$ and $1 \leq q<p<N$. problem:

For $0 \leq a<(N-p) / p, a \leq b<a+1$, and $0 \leq \mu<\bar{\mu}$, consider the following limiting

$$
\begin{aligned}
-\operatorname{div}\left(|x|^{-a p}|\nabla u|^{p-2} \nabla u\right) & -\mu \frac{|u|^{p-2} u}{|x|^{p(a+1)}}=\frac{|u|^{p^{*}(a, b)-2} u}{|x|^{b p^{*}(a, b)}} \text { in } \mathbb{R}^{N} \backslash\{0\}, \\
u & >0 \quad \text { in } \mathbb{R}^{N} \backslash\{0\}, \\
u & \in W_{a}^{1, p}\left(\mathbb{R}^{N}\right),
\end{aligned}
$$

where $W_{a}^{1, p}\left(\mathbb{R}^{N}\right)$ is the space obtained as the completion of $C_{0}^{\infty}\left(\mathbb{R}^{N}\right)$ with respect to the $\operatorname{norm}\left(\int_{\mathbb{R}^{N}}|x|^{-a p}|\nabla u|^{p} d x\right)^{1 / p}$. From [5, Lemma 2.2], we know (1.8) has a unique ground state solution $U_{p, \mu}$ satisfying

$$
U_{p, \mu}(1)=\left(\frac{p^{*}(a, b)(\bar{\mu}-\mu)}{p}\right)^{1 /\left(p^{*}(a, b)-p\right)}
$$


and all ground states must be of the form $\tilde{U}_{\varepsilon}(x)=\varepsilon^{-(N-p) / p-a} U_{p, \mu}(x / \varepsilon)$ for some $\varepsilon>0$, that is,

$$
S_{\mu, a, b}=\inf _{u \in W_{a}^{1, p}\left(\mathbb{R}^{N}\right) \backslash\{0\}} \frac{\int_{\mathbb{R}^{N}}\left(|\nabla u|^{p}-\mu|u|^{p} /|x|^{p(a+1)}\right) d x}{\left(\int_{\Omega}|u|^{p^{*}(a, b)} /|x|^{b p^{*}(a, b)} d x\right)^{p / p^{*}(a, b)}}
$$

is achieved by $\tilde{U}_{\varepsilon}$. Moreover, $U_{p, \mu}$ is radially symmetric and possesses the following properties:

$$
\begin{array}{ll}
\lim _{r \rightarrow 0} r^{\alpha(\mu)} U_{p, \mu}(r)=c_{1}>0, & \lim _{r \rightarrow 0} r^{\alpha(\mu)+1}\left|U_{p, \mu}^{\prime}(r)\right|=c_{1} \alpha(\mu) \geq 0, \\
\lim _{r \rightarrow+\infty} r^{\beta(\mu)} U_{p, \mu}(r)=c_{2}>0, & \lim _{r \rightarrow+\infty} r^{\beta(\mu)+1}\left|U_{p, \mu}^{\prime}(r)\right|=c_{2} \beta(\mu)>0,
\end{array}
$$

where $c_{i}(i=1,2)$ are positive constants and $\alpha(\mu), \beta(\mu)$ are the zeros of the function

$$
f(\tau)=(p-1) \tau^{p}-(N-p(a+1)) \tau^{p-1}+\mu, \quad \tau \geq 0,0 \leq \mu<\bar{\mu},
$$

which satisfy $0 \leq \alpha(\mu)<(N-p(a+1)) / p<\beta(\mu)<(N-p(a+1)) /(p-1)$. Furthermore, there exist the positive constants $c_{3}=c_{3}(\mu, p, a, b)$ and $c_{4}=c_{4}(\mu, p, a, b)$ such that

$$
c_{3} \leq U_{p, \mu}(x)\left(|x|^{\alpha(\mu) / \delta}+|x|^{\beta(\mu) / \delta}\right)^{\delta} \leq c_{4}, \quad \delta \triangleq \frac{N-p(a+1)}{p} .
$$

Throughout this paper, let $R_{0}$ be the positive constant such that $\Omega \subset B\left(0 ; R_{0}\right)$, where $B\left(0 ; R_{0}\right)=\left\{x \in \mathbb{R}^{N}:|x|<R_{0}\right\}$. By Hölder and Sobolev-Hardy inequalities, for all $u \in W$, we obtain

$$
\begin{aligned}
\int_{\Omega} \frac{|u|^{q}}{|x|^{d p^{*}(a, d)}} & \leq\left(\int_{B\left(0 ; R_{0}\right)}|x|^{-d p^{*}(a, d)}\right)^{\left(p^{*}(a, d)-q\right) / p^{*}(a, d)}\left(\int_{\Omega} \frac{|u|^{p^{*}(a, d)}}{|x|^{d p^{*}(a, d)}}\right)^{q / p^{*}(a, d)} \\
& \leq\left(N \omega_{N} \int_{0}^{R_{0}} r^{-d p^{*}(a, d)+N-1} d r\right)^{\left(p^{*}(a, d)-q\right) / p^{*}(a, d)}\left(S_{\mu, a, d}\right)^{-(q / p)}\|u\|^{q} \\
& \leq\left(\frac{N \omega_{N} R_{0}^{N-d p^{*}(a, d)}}{N-d p^{*}(a, d)}\right)^{\left(p^{*}(a, d)-q\right) / p^{*}(a, d)} \quad\left(S_{\mu, a, d}\right)^{-(q / p)}\|u\|^{q}
\end{aligned}
$$


Set

$$
\begin{aligned}
\Lambda_{0}= & \left(\frac{p-q}{p^{*}(a, b)-q}\right)^{(p-q) /\left(p^{*}(a, b)-p\right)}\left(\frac{p^{*}(a, b)-p}{p^{*}(a, b)-q}\right)\left(\frac{N \omega_{N} R_{0}^{N-d p^{*}(a, d)}}{N-d p^{*}(a, d)}\right)^{-\left(\left(p^{*}(a, d)-q\right) / p^{*}(a, d)\right)} \\
& \times\left(S_{\mu, a, d}\right)^{q / p}\left(S_{\mu, a, b}\right)^{p^{*}(a, b)(p-q) / p\left(p^{*}(a, b)-p\right)}
\end{aligned}
$$

where $\omega_{N}=2 \pi^{N / 2} / N \Gamma(N / 2)$ is the volume of the unit ball in $\mathbb{R}^{N}$.

Furthermore, from $0 \leq a<(N-p) / p$ and $a \leq d<a+1$, we can deduce that

$$
0<p\left(\frac{N-p}{p}-a\right)+p d=N-p(a+1-d)<N
$$

which implies

$$
\begin{gathered}
p^{*}(a, d)=\frac{p N}{N-p(a+1-d)}>p \\
N-d p^{*}(a, d)=\frac{N p}{N-p(a+1-d)}\left(\frac{N-p}{p}-a\right)>0 .
\end{gathered}
$$

Combining these with $1 \leq q<p$, we get $\Lambda_{0}>0$.

We are now ready to state our main results.

Theorem 1.1. Assume that $N \geq 3,0 \leq \mu<\bar{\mu}, 0 \leq a<(N-p) / p, a \leq b, d<a+1$, and $1 \leq q<p<N$. Then one has the following results.

(i) If $\lambda \in\left(0, \Lambda_{0}\right)$, then (1.1) has at least one positive solution in $W$.

(ii) If $\lambda \in\left(0,(q / p) \Lambda_{0}\right)$, then (1.1) has at least two positive solutions in $W$.

Remark 1.2. In [5], Kang considered (1.1) with $p$-sublinear perturbation of $p \leq q<p^{*}(a, d)$. Via variational methods, he proved the existence of positive solutions of (1.1) when the parameters $a, b, d, p, q, \lambda, \mu$ satisfy suitable conditions. But the existence of positive solutions for (1.1) involving the $p$-sublinear of $1 \leq q<p<N$ is not considered. In this paper, we will give a complement result.

This paper is organized as follows. In Sections 2 and 3, we give some preliminaries and some properties of Nehari manifold. In Section 4, we complete proofs of Theorem 1.1. At the end of this section, we explain some notations employed. In the following discussions, we will denote various positive constants as $C, C_{i}$ and omit $d x$ in the integral for convenience. We denote $B(0 ; R)$ as a ball centered at the origin with radius $R$, and $\omega_{N}=2 \pi^{N / 2} / N \Gamma(N / 2)$ is the volume of the unit ball $B(0 ; 1)$ in $\mathbb{R}^{N}$. We denote the norm in $L^{r}(\Omega)$ by $|\cdot|_{r}$ for $1 \leq r \leq \infty$, and $L^{r}\left(\Omega,|x|^{-s}\right), 1 \leq r<\infty$ is the closure of $C_{0}^{\infty}(\Omega)$ with the norm $|\cdot|_{L^{r}\left(\Omega,|x|^{-s}\right)}=\left(\int_{\Omega}|x|^{-s}|\cdot|^{r}\right)^{1 / r} \cdot W^{-1}$ denoting the dual space of $W . O\left(\varepsilon^{t}\right)$ denotes $\left|O\left(\varepsilon^{t}\right) / \varepsilon^{t}\right| \leq C$, and $o\left(\varepsilon^{t}\right)$ means $\left|o\left(\varepsilon^{t}\right) / \varepsilon^{t}\right| \rightarrow 0$ as $\varepsilon \rightarrow 0$. By o(1) we always mean it is a generic infinitesimal value. 


\section{Nehari Manifold}

Since the functional $J_{\lambda}$ is not bounded from below on $W$, we will work on Nehari manifold. For $\lambda>0$ we define

$$
\mathcal{N}_{\lambda}=\left\{u \in W \backslash\{0\}:\left\langle J_{\lambda}^{\prime}(u), u\right\rangle=0\right\}
$$

We recall that any nonzero solutions of (1.1) belong to $N_{\lambda}$. Moreover, by definition, we have that $u \in \mathcal{N}_{\curlywedge}$ if and only if

$$
\|u\| \neq 0, \quad\|u\|^{p}-\frac{1}{p^{*}(a, b)} \int_{\Omega} \frac{|u|^{p^{*}(a, b)}}{|x|^{b p^{*}(a, b)}}-\frac{\lambda}{q} \int_{\Omega} \frac{|u|^{q}}{|x|^{d p^{*}(a, d)}}=0
$$

The following result is concerned with the behavior of $J_{\lambda}$ on $N_{\lambda}$.

Lemma 2.1. $J_{\lambda}$ is coercive and bounded from below on $\boldsymbol{N}_{\lambda}$.

Proof. If $u \in \mathcal{N}_{\lambda}$, then by (1.14) and (2.2), we get

$$
\begin{aligned}
J_{\lambda}(u)= & \frac{p^{*}(a, b)-p}{p^{*}(a, b) p}\|u\|^{p}-\lambda\left(\frac{p^{*}(a, b)-q}{p^{*}(a, b) q}\right) \int_{\Omega} \frac{|u|^{q}}{|x|^{d p^{*}(a, d)}} \\
\geq & \frac{p^{*}(a, b)-p}{p^{*}(a, b) p}\|u\|^{p}-\lambda\left(\frac{p^{*}(a, b)-q}{p^{*}(a, b) q}\right)\left(\frac{N \omega_{N} R_{0}^{N-d p^{*}(a, d)}}{N-d p^{*}(a, d)}\right)^{\left(p^{*}(a, d)-q\right) / p^{*}(a, d)} \\
& \times\left(S_{\mu, a, d}\right)^{-q / p}\|u\|^{q} .
\end{aligned}
$$

Since $0 \leq a<(N-p) / p, a \leq b, d<a+1$ and $1 \leq q<p<p^{*}(a, b)$, it follows that $J_{\lambda}$ is coercive and bounded from below on $N_{\lambda}$.

Define $\psi_{\lambda}: W \rightarrow \mathbb{R}$, by $\psi_{\lambda}(u)=\left\langle J_{\lambda}^{\prime}(u), u\right\rangle$, that is,

$$
\psi_{\lambda}(u)=\|u\|^{p}-\int_{\Omega} \frac{|u|^{p^{*}(a, b)}}{|x|^{b p^{*}(a, b)}}-\lambda \int_{\Omega} \frac{|u|^{q}}{|x|^{d p^{*}(a, d)}}
$$

Note that $\psi_{\curlywedge}$ is of class $C^{1}$ with

$$
\left\langle\psi_{\lambda}^{\prime}(u), u\right\rangle=p\|u\|^{p}-p^{*}(a, b) \int_{\Omega} \frac{|u|^{p^{*}(a, b)}}{|x|^{b p^{*}(a, b)}}-q \lambda \int_{\Omega} \frac{|u|^{q}}{|x|^{d p^{*}(a, d)}}
$$


Furthermore, if $u \in \mathcal{N}_{\lambda}$, then by (2.2), we have that

$$
\begin{aligned}
\left\langle\psi_{\lambda}^{\prime}(u), u\right\rangle & =(p-q)\|u\|^{p}-\left(p^{*}(a, b)-q\right) \int_{\Omega} \frac{|u|^{p^{*}(a, b)}}{|x|^{b p^{*}(a, b)}} \\
& =\left(p-p^{*}(a, b)\right)\|u\|^{p}-\left(q-p^{*}(a, b)\right) \lambda \int_{\Omega} \frac{|u|^{q}}{|x|^{d p^{*}(a, d)}}
\end{aligned}
$$

Now we split $\mathcal{N}_{\curlywedge}$ into three sets:

$$
\begin{aligned}
& \mathcal{N}_{\lambda}^{+}=\left\{u \in \mathcal{N}_{\lambda}:\left\langle\psi_{\lambda}^{\prime}(u), u\right\rangle>0\right\}, \\
& \mathcal{N}_{\lambda}^{0}=\left\{u \in \mathcal{N}_{\lambda}:\left\langle\psi_{\lambda}^{\prime}(u), u\right\rangle=0\right\}, \\
& \mathcal{N}_{\lambda}^{-}=\left\{u \in \mathcal{N}_{\lambda}:\left\langle\psi_{\lambda}^{\prime}(u), u\right\rangle<0\right\} .
\end{aligned}
$$

$J_{\lambda}$.

The following result shows that minimizers on $\mathcal{N}_{\mathcal{\lambda}}$ are the "usual" critical points for

Lemma 2.2. Suppose $u_{0}$ is a local minimizer of $J_{\lambda}$ on $\mathcal{N}_{\lambda}$ and $u_{0} \notin \mathcal{N}_{\lambda}^{0}$. Then, $J_{\lambda}^{\prime}\left(u_{0}\right)=0$ in $W^{-1}$.

Proof. See [30, Lemma 2.2].

Motivated by the above result, we will get conditions for $\mathcal{N}_{\lambda}^{0}=\emptyset$.

Lemma 2.3. $N_{\lambda}^{0}=\emptyset$ for all $\lambda \in\left(0, \Lambda_{0}\right)$.

Proof. We argue by contradiction. Suppose that there exists a $\lambda \in\left(0, \Lambda_{0}\right)$ such that $\mathcal{N}_{\lambda}^{0} \neq \emptyset$. Let $u \in \mathcal{N}_{\lambda}^{0}$ be arbitrary, then by (2.2), (2.7), and (2.8), we have that

$$
\begin{gathered}
0<\|u\|^{p}=\frac{p^{*}(a, b)-q}{p-q} \int_{\Omega} \frac{|u|^{p^{*}(a, b)}}{|x|^{b p^{*}(a, b)}} \\
0<\|u\|^{p}=\lambda \frac{p^{*}(a, b)-q}{p^{*}(a, b)-p} \int_{\Omega} \frac{|u|^{q}}{|x|^{d p^{*}(a, d)}} .
\end{gathered}
$$

By (1.14), (2.10), and Sobolev-Hardy inequality, we get

$$
\begin{gathered}
\|u\| \geq\left(\frac{p-q}{p^{*}(a, b)-q}\right)^{1 /\left(p^{*}(a, b)-p\right)}\left(S_{\mu, a, b}\right)^{p^{*}(a, b) / p\left(p^{*}(a, b)-p\right)}, \\
\|u\| \leq\left(\lambda \frac{p^{*}(a, b)-q}{p^{*}(a, b)-p}\right)^{1 /(p-q)}\left(\frac{N \omega_{N} R_{0}^{N-d p^{*}(a, d)}}{N-d p^{*}(a, d)}\right)^{\left(p^{*}(a, d)-q\right) / p^{*}(a, d)(p-q)}\left(S_{\mu, a, d}\right)^{-(q / p(p-q))} .
\end{gathered}
$$


Hence we must have

$$
\begin{aligned}
\lambda \geq & \left(\frac{p-q}{p^{*}(a, b)-q}\right)^{(p-q) /\left(p^{*}(a, b)-p\right)}\left(\frac{p^{*}(a, b)-p}{p^{*}(a, b)-q}\right)\left(\frac{N \omega_{N} R_{0}^{N-d p^{*}(a, d)}}{N-d p^{*}(a, d)}\right)^{-\left(\left(p^{*}(a, d)-q\right) / p^{*}(a, d)\right)} \\
& \times\left(S_{\mu, a, d}\right)^{q / p}\left(S_{\mu, a, b}\right)^{p^{*}(a, b)(p-q) / p\left(p^{*}(a, b)-p\right)} \\
= & \Lambda_{0}
\end{aligned}
$$

which is a contradiction.

For each $u \in W \backslash\{0\}$, let

$$
\tau_{\max }=\left(\frac{(p-q)\|u\|^{p}}{\left(p^{*}(a, b)-q\right) \int_{\Omega}|u|^{p^{*}(a, b)} /|x|^{b p^{*}(a, b)}}\right)^{1 /\left(p^{*}(a, b)-p\right)}
$$

Lemma 2.4. If $\lambda \in\left(0, \Lambda_{0}\right)$, then for each $u \in W \backslash\{0\}$, the set $\{\tau u: \tau>0\}$ intersects $\Lambda_{\lambda}$ exactly twice. More specifically, there exist a unique $\tau^{-}=\tau^{-}(u)>0$ such that $\tau^{-} u \in \mathcal{N}_{\lambda}^{-}$and a unique $\tau^{+}=\tau^{+}(u)>0$ such that $\tau^{+} u \in \mathcal{N}_{\lambda}^{+}$. Moreover, $\tau^{+}<\tau_{\max }<\tau^{-}$and

$$
J_{\lambda}\left(\tau^{+} u\right)=\inf _{0 \leq \tau \leq \tau_{\max }} J_{\lambda}(\tau u), \quad J_{\lambda}\left(\tau^{-} u\right)=\sup _{\tau \geq \tau_{\max }} J_{\lambda}(\tau u)
$$

Proof. The proof is similar to that of [29, Lemma 2.7] and is omitted.

We remark that by Lemma 2.3 we have, $\mathcal{N}_{\lambda}=\mathcal{N}_{\lambda}^{+} \cup \mathcal{N}_{\lambda}^{-}$for all $\lambda \in\left(0, \Lambda_{0}\right)$. Furthermore, by Lemma 2.4 it follows that $\mathcal{N}_{\lambda}^{+}$and $\mathcal{N}_{\lambda}^{-}$are non-empty and by Lemma 2.1 we may define

$$
\alpha_{\lambda}=\inf _{u \in \mathcal{N}_{\curlywedge}} J_{\lambda}(u), \quad \alpha_{\lambda}^{+}=\inf _{u \in \mathcal{N}_{\lambda}^{+}} J_{\lambda}(u), \quad \alpha_{\lambda}^{-}=\inf _{u \in \mathcal{N}_{\lambda}^{-}} J_{\lambda}(u) .
$$

Theorem 2.5. $\quad$ (i) If $\lambda \in\left(0, \Lambda_{0}\right)$, then one has $\alpha_{\lambda} \leq \alpha_{\lambda}^{+}<0$.

(ii) If $\lambda \in\left(0,(q / p) \Lambda_{0}\right)$, then $\alpha_{\lambda}^{-}>d_{0}$ for some positive constant $d_{0}$.

In particular, for each $\lambda \in\left(0,(q / p) \Lambda_{0}\right)$, one has $\alpha_{\lambda}=\alpha_{\lambda}^{+}<0<\alpha_{\lambda}^{-}$.

Proof. (i) Let $u \in \mathcal{N}_{\lambda}^{+}$. By (2.7),

$$
\frac{p-q}{p^{*}(a, b)-q}\|u\|^{p}>\int_{\Omega} \frac{|u|^{p^{*}(a, b)}}{|x|^{b p^{*}(a, b)}}
$$


and so also using (2.2),

$$
\begin{aligned}
J_{\lambda}(u) & =\left(\frac{1}{p}-\frac{1}{q}\right)\|u\|^{p}+\left(\frac{1}{q}-\frac{1}{p^{*}(a, b)}\right) \int_{\Omega} \frac{|u|^{p^{*}(a, b)}}{|x|^{b p^{*}(a, b)}} \\
& <\left[\left(\frac{1}{p}-\frac{1}{q}\right)+\left(\frac{1}{q}-\frac{1}{p^{*}(a, b)}\right)\left(\frac{p-q}{p^{*}(a, b)-q}\right)\right]\|u\|^{p} \\
& =-\frac{(p-q)\left(p^{*}(a, b)-p\right)}{\left.p q p^{*}(a, b)\right)}\|u\|^{p}<0 .
\end{aligned}
$$

Therefore, from the definition of $\alpha_{\curlywedge}$ and $\alpha_{\lambda}^{+}$, we can deduce that $\alpha_{\curlywedge} \leq \alpha_{\lambda}^{+}<0$.

(ii) Let $u \in \mathcal{N}_{\lambda}^{-}$. By (2.7),

$$
\frac{p-q}{p^{*}(a, b)-q}\|u\|^{p}<\int_{\Omega} \frac{|u|^{p^{*}(a, b)}}{|x|^{b p^{*}(a, b)}} .
$$

Moreover, by Sobolev-Hardy inequality,

$$
\int_{\Omega} \frac{|u|^{p^{*}(a, b)}}{|x|^{b p^{*}(a, b)}} \leq\left(S_{\mu, a, b}\right)^{-\left(p^{*}(a, b) / p\right)}\|u\|^{p^{*}(a, b)} .
$$

This implies

$$
\|u\|>\left(\frac{p-q}{p^{*}(a, b)-q}\right)^{1 /\left(p^{*}(a, b)-p\right)}\left(S_{\mu, a, b}\right)^{p^{*}(a, b) / p\left(p^{*}(a, b)-p\right)} \quad \forall u \in \mathcal{N}_{\lambda}^{-} .
$$

By (2.4) and (2.20), we have

$$
\begin{aligned}
J_{\lambda}(u) \geq & \|u\|^{q}\left[\frac{p^{*}(a, b)-p}{p^{*}(a, b) p}\|u\|^{p-q}-\lambda\left(\frac{p^{*}(a, b)-q}{p^{*}(a, b) q}\right)\right. \\
& \left.\times\left(\frac{N \omega_{N} R_{0}^{N-d p^{*}(a, d)}}{N-d p^{*}(a, d)}\right)^{\left(p^{*}(a, d)-q\right) / p^{*}(a, d)}\left(S_{\mu, a, d}\right)^{-(q / p)}\right] \\
> & \left(\frac{p-q}{p^{*}(a, b)-q}\right)^{q /\left(p^{*}(a, b)-p\right)}\left(S_{\mu, a, b}\right)^{q p^{*}(a, b) / p\left(p^{*}(a, b)-p\right)} \\
\times & {\left[\frac{p^{*}(a, b)-p}{p^{*}(a, b) p}\left(\frac{p-q}{p^{*}(a, b)-q}\right)^{(p-q) /\left(p^{*}(a, b)-p\right)}\left(S_{\mu, a, b}\right)^{p^{*}(a, b)(p-q) / p\left(p^{*}(a, b)-p\right)}\right.} \\
& -\lambda\left(\frac{p^{*}(a, b)-q}{p^{*}(a, b) q}\right)\left(\frac{N \omega_{N} R_{0}^{N-d p^{*}(a, d)}}{N-d p^{*}(a, d)}\right)^{\left(p^{*}(a, d)-q\right) / p^{*}(a, d)}
\end{aligned}
$$




$$
\begin{aligned}
= & \left(\frac{q}{p} \Lambda_{0}-\lambda\right)\left(\frac{p-q}{p^{*}(a, b)-q}\right)^{q /\left(p^{*}(a, b)-p\right)}\left(S_{\mu, a, b}\right)^{q p^{*}(a, b) / p\left(p^{*}(a, b)-p\right)}\left(\frac{p^{*}(a, b)-q}{p^{*}(a, b) q}\right) \\
& \times\left(\frac{N \omega_{N} R_{0}^{N-d p^{*}(a, d)}}{N-d p^{*}(a, d)}\right)^{\left(p^{*}(a, d)-q\right) / p^{*}(a, d)}\left(S_{\mu, a, d}\right)^{-(q / p)} .
\end{aligned}
$$

Thus, if $\lambda \in\left(0,(q / p) \Lambda_{0}\right)$, then

$$
J_{\lambda}(u)>d_{0} \quad \forall u \in \mathcal{N}_{\lambda}^{-}
$$

for some positive constant $d_{0}$.

Remark 2.6. If $\lambda \in\left(0,(q / p) \Lambda_{0}\right)$, then by Lemma 2.4 and Theorem 2.5 , for each $u \in W \backslash\{0\}$, we can easily deduce that

$$
\tau^{-} u \in \mathcal{N}_{\lambda^{\prime}}^{-} \quad J_{\lambda}\left(\tau^{-} u\right)=\sup _{\tau \geq 0} J_{\lambda}(\tau u) \geq \alpha_{\lambda}^{-}>0
$$

\section{Proof of the Main Results}

First, we define the Palais-Smale (simply by (PS)) sequences, (PS)-values, and (PS)conditions in $W$ for $J_{\lambda}$ as follows.

Definition 3.1. (i) For $c \in \mathbb{R}$, a sequence $\left\{u_{n}\right\}$ is a (PS) $)_{c}$-sequence in $W$ for $J_{\lambda}$ if $J_{\lambda}\left(u_{n}\right)=c+o(1)$ and $J_{\lambda}^{\prime}\left(u_{n}\right)=o(1)$ strongly in $W^{-1}$ as $n \rightarrow \infty$.

(ii) $c \in \mathbb{R}$ is a (PS)-value in $W$ for $J_{\lambda}$ if there exists a (PS) $)_{c}$-sequence in $W$ for $J_{\lambda}$.

(iii) $J_{\lambda}$ satisfies the (PS) $)_{c}$-condition in $W$ if any (PS) $)_{c}$-sequence $\left\{u_{n}\right\}$ in $W$ for $J_{\lambda}$ contains a convergent subsequence.

Now, we use the Ekeland variational principle [35] to get the following results.

Proposition 3.2. (i) If $\lambda \in\left(0, \Lambda_{0}\right)$, then $J_{\lambda}$ has a $(P S)_{\alpha_{\lambda}}$-sequence $\left\{u_{n}\right\} \subset \mathcal{N}_{\lambda}$.

(ii) If $\lambda \in\left(0,(q / p) \Lambda_{0}\right)$, then $J_{\lambda}$ has a $(P S)_{\alpha_{\lambda}^{-}}$sequence $\left\{u_{n}\right\} \subset \mathcal{N}_{\lambda^{-}}$.

Proof. The proof is similar to [29, Proposition 3.3] and the details are omitted.

Now, we establish the existence of a local minimum for $J_{\lambda}$ on $N_{\lambda}$.

Theorem 3.3. Assume that $N \geq 3,0 \leq \mu<\bar{\mu}, 0 \leq a<(N-p) / p, a \leq b, d<a+1$, and $1 \leq q<p<N$. If $\lambda \in\left(0, \Lambda_{0}\right)$, then there exists $u_{\lambda} \in \mathcal{N}_{\lambda}^{+}$such that

(i) $J_{\lambda}\left(u_{\curlywedge}\right)=\alpha_{\curlywedge}=\alpha_{\lambda}^{+}$

(ii) $u_{\curlywedge}$ is a positive solution of (1.1),

(iii) $\left\|u_{\lambda}\right\| \rightarrow 0$ as $\lambda \rightarrow 0^{+}$. 
Proof. By Proposition 3.2(i), there exists a minimizing sequence $\left\{u_{n}\right\} \subset \mathcal{N}_{\curlywedge}$ such that

$$
J_{\lambda}\left(u_{n}\right)=\alpha_{\lambda}+o(1), \quad J_{\lambda}^{\prime}\left(u_{n}\right)=o(1) \quad \text { in } W^{-1} .
$$

Since $J_{\lambda}$ is coercive on $N_{\lambda}$ (see Lemma 2.1), we get that $\left\{u_{n}\right\}$ is bounded in $W$. From [5, Lemma 2.1], we deduce that the embedding $W \hookrightarrow L^{r}\left(\Omega,|x|^{-d p^{*}(a, d)}\right)$ is compact for $1 \leq r<p^{*}(a, d)$. Thus, there exists $u_{\lambda} \in W$, passing to a subsequence if necessary, using similar arguments found in $[27,36]$, then one can get that as $n \rightarrow \infty$

$$
\begin{aligned}
& u_{n} \rightarrow u_{\lambda} \text { weakly in } W, \\
& u_{n} \longrightarrow u_{\curlywedge} \text { strongly in } L^{q}\left(\Omega,|x|^{-d p^{*}(a, d)}\right) \quad \text { for } 1 \leq q<p, \\
& u_{n} \longrightarrow u_{\lambda} \text { a.e. in } \Omega, \\
& \nabla u_{n} \longrightarrow \nabla u_{\lambda} \text { a.e. in } \Omega, \\
& \frac{u_{n}}{|x|^{a+1}} \rightarrow \frac{u_{\curlywedge}}{|x|^{a+1}} \text { weakly in } L^{p}(\Omega), \\
& \int_{\Omega} \frac{\left|u_{n}\right|^{p^{*}(a, b)-2} u_{n}}{|x|^{b p^{*}(a, b)}} v \longrightarrow \int_{\Omega} \frac{\left|u_{\curlywedge}\right|^{p^{*}(a, b)-2} u_{\curlywedge}}{|x|^{b p^{*}(a, b)}} v, \quad \forall v \in W .
\end{aligned}
$$

Consequently, passing to the limit in $\left\langle J_{\lambda}^{\prime}\left(u_{n}\right), v\right\rangle$, by (3.1) and (3.2), as $n \rightarrow \infty$, we have

$$
\int_{\Omega}\left(\frac{\left|\nabla u_{\lambda}\right|^{p-2} \nabla u_{\lambda} \nabla v}{|x|^{a p}}-\mu \frac{\left|u_{\lambda}\right|^{p-2} u_{\curlywedge} v}{|x|^{p(a+1)}}\right)-\int_{\Omega} \frac{\left|u_{\lambda}\right|^{p^{*}(a, b)-2} u_{\lambda} v}{|x|^{b p^{*}(a, b)}}-\lambda \int_{\Omega} \frac{\left|u_{\lambda}\right|^{q-2} u_{\lambda} v}{|x|^{d p^{*}(a, d)}}=0
$$

for all $v \in W$. That is, $\left\langle J_{\lambda}^{\prime}\left(u_{\curlywedge}\right), v\right\rangle=0$. Thus $u_{\curlywedge}$ is a weak solution of (1.1). Furthermore, from $u_{n} \in \mathcal{N}_{\Lambda}$ and (2.3), we deduce that

$$
\lambda \int_{\Omega} \frac{\left|u_{n}\right|^{q}}{|x|^{d p^{*}(a, d)}}=\frac{q\left(p^{*}(a, b)-p\right)}{p\left(p^{*}(a, b)-q\right)}\left\|u_{n}\right\|^{p}-\frac{p^{*}(a, b) q}{p^{*}(a, b)-q} J_{\lambda}\left(u_{n}\right)
$$

Let $n \rightarrow \infty$ in (3.4), by (3.1) and (3.2), and since $\alpha_{\lambda}<0$ by (i) of Theorem 2.5, we get

$$
\lambda \int_{\Omega} \frac{\left|u_{\lambda}\right|^{q}}{|x|^{d p^{*}(a, d)}} \geq-\frac{p^{*}(a, b) q}{p^{*}(a, b)-q} \alpha_{\curlywedge}>0
$$

Thus $u_{\lambda} \not \equiv 0$, and since $J_{\lambda}^{\prime}\left(u_{\lambda}\right)=0$, it follows that $u_{\lambda} \in \mathcal{N}_{\lambda}$ and, in particular, $J_{\lambda}\left(u_{\lambda}\right) \geq \alpha_{\lambda}$. 
Next, we will show, up to a subsequence, that $u_{n} \rightarrow u_{\lambda}$ strongly in $W$ and $J_{\lambda}\left(u_{\lambda}\right)=\alpha_{\lambda}$. From the fact $u_{n}, u \in \mathcal{N}_{\lambda},(2.3)$ and the Fatou's lemma, it follows that

$$
\begin{aligned}
\alpha_{\lambda} & \leq J_{\lambda}\left(u_{\lambda}\right)=\frac{p^{*}(a, b)-p}{p p^{*}(a, b)}\left\|u_{\lambda}\right\|^{p}-\lambda\left(\frac{p^{*}(a, b)-q}{p^{*}(a, b) q}\right) \int_{\Omega} \frac{\left|u_{\lambda}\right|^{q}}{|x|^{d p^{*}(a, d)}} \\
& \leq \liminf _{n \rightarrow \infty}\left[\frac{p^{*}(a, b)-p}{p^{*}(a, b) p}\left\|u_{n}\right\|^{p}-\lambda\left(\frac{p^{*}(a, b)-q}{p^{*}(a, b) q}\right) \int_{\Omega} \frac{\left|u_{n}\right|^{q}}{|x|^{d p^{*}(a, d)}}\right] \\
& =\liminf _{n \rightarrow \infty} J_{\lambda}\left(u_{n}\right)=\alpha_{\lambda},
\end{aligned}
$$

which implies that $J_{\lambda}\left(u_{\lambda}\right)=\alpha_{\lambda}$ and $\lim _{n \rightarrow \infty}\left\|u_{n}\right\|^{p}=\left\|u_{\lambda}\right\|^{p}$. Standard argument shows that $u_{n} \rightarrow u_{\lambda}$ strongly in $W$. Moreover, $u_{\lambda} \in \mathcal{N}_{\lambda}^{+}$. Otherwise, if $u_{\lambda} \in \mathcal{N}_{\lambda}^{-}$, by Lemma 2.4, there exist unique $\tau_{\lambda}^{+}$and $\tau_{\lambda}^{-}$such that $\tau_{\lambda}^{+} u_{\lambda} \in \mathcal{N}_{\lambda}^{+}, \tau_{\lambda}^{-} u_{\lambda} \in \mathcal{N}_{\lambda}^{-}$and $\tau_{\lambda}^{+}<\tau_{\lambda}^{-}=1$. Since

$$
\frac{d}{d \tau} J_{\lambda}\left(\tau_{\lambda}^{+} u_{\curlywedge}\right)=0, \quad \frac{d^{2}}{d \tau^{2}} J_{\lambda}\left(\tau_{\lambda}^{+} u_{\lambda}\right)>0
$$

there exists $\bar{\tau} \in\left(\tau_{\lambda}^{+}, \tau_{\lambda}^{-}\right)$such that $J_{\lambda}\left(\tau_{\lambda}^{+} u_{\lambda}\right)<J_{\lambda}\left(\bar{\tau} u_{\lambda}\right)$. By Lemma 2.4 we get that

$$
J_{\lambda}\left(\tau_{\lambda}^{+} u_{\lambda}\right)<J_{\lambda}\left(\bar{\tau} u_{\lambda}\right) \leq J_{\lambda}\left(\tau_{\lambda}^{-} u_{\lambda}\right)=J_{\lambda}\left(u_{\lambda}\right)
$$

which is a contradiction. Since $J_{\lambda}\left(u_{\lambda}\right)=J_{\lambda}\left(\left|u_{\lambda}\right|\right)$ and $\left|u_{\lambda}\right| \in \mathcal{N}_{\lambda}^{+}$, by Lemma 2.2, we may assume that $u_{\lambda}$ is a nontrivial nonnegative solution of (1.1). By [5, Lemma 2.3], it follows that $u_{\lambda}>0$ in $\Omega$. Finally, by (1.14) and (2.8), we obtain

$$
\left\|u_{\lambda}\right\|^{p-q}<\lambda\left(\frac{p^{*}(a, b)-q}{p^{*}(a, b)-p}\right)\left(\frac{N \omega_{N} R_{0}^{N-d p^{*}(a, d)}}{N-d p^{*}(a, d)}\right)^{\left(p^{*}(a, d)-q\right) / p^{*}(a, d)}\left(S_{\mu, a, d}\right)^{-(q / p)}
$$

which implies that $\left\|u_{\lambda}\right\| \rightarrow 0$ as $\lambda \rightarrow 0^{+}$.

Next, we will establish the existence of the second positive solution of (1.1) by proving that $J_{\lambda}$ satisfies the (PS) $\alpha_{\lambda}^{- \text {-condition. }}$

Lemma 3.4. Let $\left\{u_{n}\right\}$ be a bounded sequence in $W$. If $\left\{u_{n}\right\}$ is a $(P S)_{c}$-sequence for $J_{\lambda}$ with

$$
c \in\left(0, \frac{p^{*}(a, b)-p}{p^{*}(a, b) p}\left(S_{\mu, a, b}\right)^{p^{*}(a, b) /\left(p^{*}(a, b)-p\right)}\right)
$$

then there exists a subsequence of $\left\{u_{n}\right\}$ converging weakly to a nonzero solution of (1.1).

Proof. Let $\left\{u_{n}\right\} \subset W$ be a (PS) $)_{c}$-sequence for $J_{\lambda}$ with $c \in\left(0,\left(\left(p^{*}(a, b)-\right.\right.\right.$ $\left.\left.p) / p^{*}(a, b) p\right)\left(S_{\mu, a, b}\right)^{p^{*}(a, b) /\left(p^{*}(a, b)-p\right)}\right)$. Since $\left\{u_{n}\right\}$ is bounded in $W$ and the embedding 
$W \hookrightarrow L^{r}\left(\Omega,|x|^{-d p^{*}(a, d)}\right)$ is compact for $1 \leq r<p^{*}(a, d)$ (see [5, Lemma 2.1]), thus passing to a subsequence if necessary, we may assume that as $n \rightarrow \infty$

$$
\begin{aligned}
& u_{n} \rightarrow u_{0} \text { weakly in } W, \\
& u_{n} \rightarrow u_{0} \text { weakly in } L^{p^{*}(a, b)}\left(\Omega,|x|^{-b p^{*}(a, b)}\right) \\
& u_{n} \longrightarrow u_{0} \text { strongly in } L^{q}\left(\Omega,|x|^{-d p^{*}(a, d)}\right) \text { for } 1 \leq q<p, \\
& u_{n} \longrightarrow u_{0} \text { a.e. in } \Omega .
\end{aligned}
$$

Using the same argument in Theorem 3.3, we deduce that $J_{\lambda}^{\prime}\left(u_{0}\right)=0$ and

$$
\lambda \int_{\Omega} \frac{\left|u_{n}\right|^{q}}{|x|^{d p^{*}(a, d)}}=\lambda \int_{\Omega} \frac{\left|u_{0}\right|^{q}}{|x|^{d p^{*}(a, d)}}+o(1)
$$

Next we verify that $u_{0} \not \equiv 0$. Arguing by contradiction, we assume $u_{0} \equiv 0$. Set

$$
l=\lim _{n \rightarrow \infty} \int_{\Omega} \frac{\left|u_{n}\right|^{p^{*}(a, b)}}{|x|^{p^{*}(a, b)}} .
$$

Since $J_{\lambda}^{\prime}\left(u_{0}\right)=0$ and $\left\{u_{n}\right\}$ is bounded in $W$, then by (3.12), we can deduce that

$$
0=\left\langle\lim _{n \rightarrow \infty} J_{\lambda}^{\prime}\left(u_{n}\right), u_{n}\right\rangle=\lim _{n \rightarrow \infty}\left(\left\|u_{n}\right\|^{p}-\int_{\Omega} \frac{\left|u_{n}\right|^{p^{*}(a, b)}}{|x|^{b p^{*}(a, b)}}\right)=\lim _{n \rightarrow \infty}\left\|u_{n}\right\|^{p}-l,
$$

that is,

$$
\lim _{n \rightarrow \infty}\left\|u_{n}\right\|^{p}=l
$$

If $l=0$, then by (3.12)-(3.15), we get

$$
c=\lim _{n \rightarrow \infty} J_{\lambda}\left(u_{n}\right)=\lim _{n \rightarrow \infty}\left(\frac{1}{p}\left\|u_{n}\right\|^{p}-\int_{\Omega} \frac{\left|u_{n}\right|^{p^{*}(a, b)}}{|x|^{b p^{*}(a, b)}}-\lambda \int_{\Omega} \frac{\left|u_{n}\right|^{q}}{|x|^{d p^{*}(a, d)}}\right)=0,
$$

which contradicts $c>0$. Thus we conclude that $l>0$. Furthermore, the Sobolev-Hardy inequality implies that

$$
\left\|u_{n}\right\|^{p} \geq S_{\mu, a, b}\left(\int_{\Omega} \frac{\left|u_{n}\right|^{p^{*}(a, b)}}{|x|^{b p^{*}(a, b)}}\right)^{p / p^{*}(a, b)}
$$

Then as $n \rightarrow \infty$, we have $l=\lim _{n \rightarrow \infty}\left\|u_{n}\right\|^{p} \geq S_{\mu, a, b} b^{p / p^{*}(a, b)}$, which implies that

$$
l \geq\left(S_{\mu, a, b}\right)^{p^{*}(a, b) /\left(p^{*}(a, b)-p\right)} .
$$


Hence, from (3.12)-(3.18) we get

$$
\begin{aligned}
c & =\lim _{n \rightarrow \infty} J_{\lambda}\left(u_{n}\right) \\
& =\frac{1}{p} \lim _{n \rightarrow \infty}\left\|u_{n}\right\|^{p}-\frac{1}{p^{*}(a, b)} \lim _{n \rightarrow \infty} \int_{\Omega} \frac{\left|u_{n}\right|^{p^{*}(a, b)}}{|x|^{b p^{*}(a, b)}}-\frac{\lambda}{q} \lim _{n \rightarrow \infty} \int_{\Omega} \frac{\left|u_{n}\right|^{q}}{|x|^{d p^{*}(a, d)}} \\
& =\left(\frac{1}{p}-\frac{1}{p^{*}(a, b)}\right) l \\
& \geq \frac{p^{*}(a, b)-p}{p^{*}(a, b) p}\left(S_{\mu, a, b}\right)^{p^{*}(a, b) /\left(p^{*}(a, b)-p\right)} .
\end{aligned}
$$

This contradicts the definition of $c$. Therefore, $u_{0}$ is a nontrivial solution of (1.1).

Lemma 3.5. If $N \geq 3,0 \leq \mu<\bar{\mu}, 0 \leq a<(N-p) / p, a \leq b, d<a+1$, and $1 \leq q<p<N$, then for any $\lambda>0$, there exists $v_{\lambda} \in W$ such that

$$
\sup _{\tau \geq 0} J_{\lambda}\left(\tau v_{\lambda}\right)<\frac{p^{*}(a, b)-p}{p^{*}(a, b) p}\left(S_{\mu, a, b}\right)^{p^{*}(a, b) /\left(p^{*}(a, b)-p\right)} .
$$

In particular, $\alpha_{\lambda}^{-}<\left(\left(p^{*}(a, b)-p\right) / p^{*}(a, b) p\right)\left(S_{\mu, a, b}\right)^{p^{*}(a, b) /\left(p^{*}(a, b)-p\right)}$ for all $\lambda \in\left(0, \Lambda_{0}\right)$.

Proof. Let $U_{p, \mu}$ be a ground state solution of (1.8), $\rho>0$ small enough such that $B(0 ; \rho) \subset$ $\Omega, \eta \in C_{0}^{\infty}(\Omega), 0 \leq \eta(x) \leq 1, \eta(x)=1$ for $|x|<\rho / 2, \eta(x)=0$ for $|x| \geq \rho$. Set $\tilde{U}_{\varepsilon}(x)=$ $\varepsilon^{-((N-p) / p)-a} U_{p, \mu}(x / \varepsilon)$ and $u_{\varepsilon}(x)=\eta(x) \tilde{U}_{\varepsilon}(x), \varepsilon>0$. Then, following the same lines as in [5], we get the following estimates as $\varepsilon \rightarrow 0$ :

$$
\begin{gathered}
\left\|u_{\varepsilon}\right\|^{p}=\left(S_{\mu, a, b}\right)^{p^{*}(a, b) /\left(p^{*}(a, b)-p\right)}+O\left(\varepsilon^{\beta(\mu) p+p(a+1)-N}\right), \\
\int_{\Omega} \frac{\left|u_{\varepsilon}\right|^{p^{*}(a, b)}}{|x|^{b p^{*}(a, b)}}=\left(S_{\mu, a, b}\right)^{p^{*}(a, b) /\left(p^{*}(a, b)-p\right)}+O\left(\varepsilon^{(\beta(\mu)+b) p^{*}(a, b)-N}\right), \\
\int_{\Omega} \frac{\left|u_{\varepsilon}\right|^{q}}{|x|^{d p^{*}(a, d)}} \geq \begin{cases}C \varepsilon^{N-d p^{*}(a, d)-q \delta}, & \frac{N-d p^{*}(a, d)}{\beta(\mu)}<q<p^{*}(a, d), \\
C \varepsilon^{q(\beta(\mu)-\delta)}|\ln \varepsilon|, & q=\frac{N-d p^{*}(a, d)}{\beta(\mu)}, \\
C \varepsilon^{q(\beta(\mu)-\delta)}, & 1 \leq q<\frac{N-d p^{*}(a, d)}{\beta(\mu)},\end{cases}
\end{gathered}
$$

where $b(\mu)$ is given in the introduction satisfying $\delta=(N-p(a+1)) / p<b(\mu)<(N-p(a+$ $1)) /(p-1)$. 
Now we consider the following functions:

$$
\begin{gathered}
g(\tau)=J_{\lambda}\left(\tau u_{\varepsilon}\right)=\frac{\tau^{p}}{p}\left\|u_{\varepsilon}\right\|^{p}-\frac{\tau^{p^{*}(a, b)}}{p^{*}(a, b)} \int_{\Omega} \frac{\left|u_{\varepsilon}\right|^{p^{*}(a, b)}}{|x|^{b p^{*}(a, b)}}-\lambda \frac{\tau^{q}}{q} \int_{\Omega} \frac{\left|u_{\varepsilon}\right|^{q}}{|x|^{d p^{*}(a, d)}} \\
\bar{g}(\tau)=\frac{\tau^{p}}{p}\left\|u_{\varepsilon}\right\|^{p}-\frac{\tau^{p^{*}(a, b)}}{p^{*}(a, b)} \int_{\Omega} \frac{\left|u_{\varepsilon}\right|^{p^{*}(a, b)}}{|x|^{b p^{*}(a, b)}}
\end{gathered}
$$

Using the definitions of $g$ and $u_{\varepsilon}$, we get

$$
g(\tau)=J_{\lambda}\left(\tau u_{\varepsilon}\right) \leq \frac{\tau^{p}}{p}\left\|u_{\varepsilon}\right\|^{p}, \quad \forall \tau \geq 0, \lambda>0
$$

Combining this with (3.21), let $\varepsilon \in(0,1)$, then there exists $\tau_{0} \in(0,1)$ independent of $\varepsilon$ such that

$$
\sup _{0 \leq \tau \leq \tau_{0}} g(\tau)<\frac{p^{*}(a, b)-p}{p^{*}(a, b) p}\left(S_{\mu, a, b}\right)^{p^{*}(a, b) /\left(p^{*}(a, b)-p\right)}, \quad \forall \lambda>0, \forall \varepsilon \in(0,1)
$$

On the other hand, by the fact for $B_{1}, B_{2}>0$

$$
\max _{\tau \geq 0}\left(\frac{\tau^{p}}{p} B_{1}-\frac{\tau^{p^{*}(a, b)}}{p^{*}(a, b)} B_{2}\right)=\frac{p^{*}(a, b)-p}{p^{*}(a, b) p}\left(B_{1}\right)^{p^{*}(a, b) /\left(p^{*}(a, b)-p\right)}\left(B_{2}\right)^{-\left(p /\left(p^{*}(a, b)-p\right)\right)}
$$

and by (3.21) and (3.22), we can get that

$$
\begin{aligned}
\max _{\tau \geq 0} \bar{g}(\tau)= & \frac{p^{*}(a, b)-p}{p^{*}(a, b) p}\left\|u_{\varepsilon}\right\|^{p^{*}(a, b) /\left(p^{*}(a, b)-p\right)}\left(\int_{\Omega} \frac{\left|u_{\varepsilon}\right|^{p^{*}(a, b)}}{|x|^{p^{*}(a, b)}}\right)^{-\left(p /\left(p^{*}(a, b)-p\right)\right)} \\
= & \frac{p^{*}(a, b)-p}{p^{*}(a, b) p}\left(\left(S_{\mu, a, b}\right)^{p^{*}(a, b) /\left(p^{*}(a, b)-p\right)}+O\left(\varepsilon^{\beta(\mu) p+p(a+1)-N}\right)^{p^{*}(a, b) /\left(p^{*}(a, b)-p\right)}\right. \\
& \times\left(\left(S_{\mu, a, b}\right)^{p^{*}(a, b) /\left(p^{*}(a, b)-p\right)}+O\left(\varepsilon^{(\beta(\mu)+b) p^{*}(a, b)-N}\right)\right)^{-\left(p /\left(p^{*}(a, b)-p\right)\right)} \\
= & \frac{p^{*}(a, b)-p}{p^{*}(a, b) p}\left(S_{\mu, a, b}\right)^{p^{*}(a, b) /\left(p^{*}(a, b)-p\right)}+O\left(\varepsilon^{\beta(\mu) p+p(a+1)-N}\right) .
\end{aligned}
$$

Hence as $\lambda>0,1 \leq q<p$, by (3.28) we have that

$$
\begin{aligned}
\sup _{\tau \geq \tau_{0}} g(\tau) & =\sup _{\tau \geq \tau_{0}}\left(\bar{g}(\tau)-\lambda \frac{\tau^{q}}{q} \int_{\Omega} \frac{\left|u_{\varepsilon}\right|^{q}}{|x|^{d p^{*}(a, d)}}\right) \\
& \leq \frac{p^{*}(a, b)-p}{p^{*}(a, b) p}\left(S_{\mu, a, b}\right)^{p^{*}(a, b) /\left(p^{*}(a, b)-p\right)}+O\left(\varepsilon^{\beta(\mu) p+p(a+1)-N}\right)-\lambda \frac{\tau_{0}^{q}}{q} \int_{\Omega} \frac{\left|u_{\varepsilon}\right|^{q}}{|x|^{d p^{*}(a, d)}} .
\end{aligned}
$$


(i) If $1 \leq q<\left(N-d p^{*}(a, d)\right) / \beta(\mu)$, then by (3.23) we have that

$$
\int_{\Omega} \frac{\left|u_{\varepsilon}\right|^{q}}{|x|^{d p^{*}(a, d)}} \geq C \varepsilon^{q(\beta(\mu)-\delta)}
$$

and since $b(\mu)>\delta=(N-p(a+1)) / p$, then

$$
\beta(\mu) p+p(a+1)-N=p(\beta(\mu)-\delta)>q(\beta(\mu)-\delta) .
$$

Combining this with (3.26) and (3.29), for any $\lambda>0$, we can choose $\varepsilon_{\lambda}$ small enough such that

$$
\sup _{\tau \geq 0} J_{\lambda}\left(\tau u_{\varepsilon_{\lambda}}\right)<\frac{p^{*}(a, b)-p}{p^{*}(a, b) p}\left(S_{\mu, a, b}\right)^{p^{*}(a, b) /\left(p^{*}(a, b)-p\right)} .
$$

(ii) If $\left(N-d p^{*}(a, d)\right) / \beta(\mu) \leq q<p$, then by (3.23) and $b(\mu)>\delta=(N-p(a+1)) / p$ we have that

$$
\begin{gathered}
\int_{\Omega} \frac{\left|u_{\varepsilon}\right|^{q}}{|x|^{d p^{*}(a, d)}} \geq \begin{cases}C \varepsilon^{N-d p^{*}(a, d)-q \delta}, & \frac{N-d p^{*}(a, d)}{\beta(\mu)}<q<p^{*}(a, d), \\
C \varepsilon^{q(\beta(\mu)-\delta)}|\ln \varepsilon|, & q=\frac{N-d p^{*}(a, d)}{\beta(\mu)},\end{cases} \\
N-d p^{*}(a, d)-q \delta \leq q \beta(\mu)-q \delta<p(\beta(\mu)-\delta)=\beta(\mu) p+p(a+1)-N .
\end{gathered}
$$

Combining this with (3.26) and (3.29), for any $\lambda>0$, we can choose $\varepsilon_{\mathcal{\lambda}}$ small enough such that

$$
\sup _{\tau \geq 0} J_{\lambda}\left(\tau u_{\varepsilon_{\curlywedge}}\right)<\frac{p^{*}(a, b)-p}{p^{*}(a, b) p}\left(S_{\mu, a, b}\right)^{p^{*}(a, b) /\left(p^{*}(a, b)-p\right)}
$$

From (i) and (ii), (3.20) holds by taking $v_{\lambda}=u_{\varepsilon_{\lambda}}$.

From Lemma 2.4, the definition of $\alpha_{\lambda}^{-}$, and (3.20), for any $\lambda \in\left(0, \Lambda_{0}\right)$, we obtain that there exists $\tau_{\lambda}^{-}>0$ such that $\tau_{\lambda}^{-} v_{\lambda} \in \mathcal{N}_{\lambda}^{-}$and

$$
\alpha_{\lambda}^{-} \leq J_{\lambda}\left(\tau_{\lambda}^{-} v_{\lambda}\right) \leq \sup _{t \geq 0} J_{\lambda}\left(\tau v_{\lambda}\right)<\frac{p^{*}(a, b)-p}{p^{*}(a, b) p}\left(S_{\mu, a, b}\right)^{p^{*}(a, b) /\left(p^{*}(a, b)-p\right)} .
$$

The proof is thus complete.

Now, we establish the existence of a local minimum of $J_{\lambda}$ on $\mathcal{N}_{\lambda}^{-}$.

Theorem 3.6. Assume that $N \geq 3,0 \leq \mu<\bar{\mu}, 0 \leq a<(N-p) / p, a \leq b, d<a+1$ and $1 \leq q<p<N$. If $\lambda \in\left(0,(q / p) \Lambda_{0}\right)$, then there exists $U_{\lambda} \in \mathcal{N}_{\lambda}^{-}$such that

(i) $J_{\lambda}\left(U_{\lambda}\right)=\alpha_{\lambda^{\prime}}^{-}$

(ii) $U_{\curlywedge}$ is a positive solution of (1.1). 
Proof. If $\lambda \in\left(0,(q / p) \Lambda_{0}\right)$, then by Theorem 2.5 (ii), Proposition 3.2 (ii), and Lemma 3.5, there exists a (PS) $\alpha_{\lambda}^{-}$-sequence $\left\{u_{n}\right\} \quad \subset \mathcal{N}_{\lambda}^{-}$in $W$ for $J_{\lambda}$ with $\alpha_{\lambda}^{-} \in\left(0,\left(\left(p^{*}(a, b)-\right.\right.\right.$ $\left.\left.p) / p^{*}(a, b) p\right)\left(S_{\mu, a, b}\right)^{p^{*}(a, b) /\left(p^{*}(a, b)-p\right)}\right)$. Since $J_{\lambda}$ is coercive on $\mathcal{N}_{\lambda}$ (see Lemma 2.1), we get that $\left\{u_{n}\right\}$ is bounded in $W$. From Lemma 3.4, there exists a subsequence still denoted by $\left\{u_{n}\right\}$ and a nontrivial solution $U_{\lambda} \in W$ of (1.1) such that $u_{n} \rightarrow U_{\curlywedge}$ weakly in $W$.

First, we prove that $U_{\lambda} \in \mathcal{N}_{\lambda}^{-}$. Arguing by contradiction, we assume $U_{\lambda} \in \mathcal{N}_{\lambda}^{+}$. Since $\mathcal{N}_{\lambda}^{-}$is closed in $W$, we have $\left\|U_{\lambda}\right\|<\liminf _{n \rightarrow \infty}\left\|u_{n}\right\|$. Thus, by Lemma 2.4, there exists a unique $\tau_{\lambda}^{-}$such that $\tau_{\lambda}^{-} U_{\lambda} \in \mathcal{N}_{\lambda}^{-}$. If $u \in N_{\lambda}$, then it is easy to see that

$$
J_{\lambda}(u)=\frac{p^{*}(a, b)-p}{p^{*}(a, b) p}\|u\|^{p}-\lambda\left(\frac{p^{*}(a, b)-q}{p^{*}(a, b) q}\right) \int_{\Omega} \frac{|u|^{q}}{|x|^{d p^{*}(a, d)}}
$$

From Remark 2.6, $u_{n} \in \mathcal{N}_{\lambda}^{-},\left\|U_{\lambda}\right\|<\lim _{\inf _{n \rightarrow \infty}}\left\|u_{n}\right\|$, and (3.36), we can deduce that

$$
\alpha_{\lambda}^{-} \leq J_{\lambda}\left(\tau_{\lambda}^{-} U_{\lambda}\right)<\lim _{n \rightarrow \infty} J_{\lambda}\left(\tau_{\lambda}^{-} u_{n}\right) \leq \lim _{n \rightarrow \infty} J_{\lambda}\left(u_{n}\right)=\alpha_{\lambda}^{-}
$$

This is a contradiction. Thus, $U_{\lambda} \in \mathcal{N}_{\lambda}^{-}$.

Next, by the same argument as that in Theorem 3.3, we get that $u_{n} \rightarrow U_{\lambda}$ strongly in $W$ and $J_{\lambda}\left(U_{\lambda}\right)=\alpha_{\lambda}^{-}>0$ for all $\lambda \in\left(0,(q / p) \Lambda_{0}\right)$. Since $J_{\lambda}\left(U_{\lambda}\right)=J_{\lambda}\left(\left|U_{\lambda}\right|\right)$ and $\left|U_{\lambda}\right| \in \mathcal{N}_{\lambda}^{-}$, by Lemma 2.2, we may assume that $U_{\lambda}$ is a nontrivial nonnegative solution of (1.1). Finally, by [5, Lemma 2.3], we obtain that $U_{\lambda}$ is a positive solution of (1.1).

Now, we complete the proof of Theorem 1.1. The part (i) of Theorem 1.1 immediately follows from Theorem 3.3. When $0<\lambda<(q / p) \Lambda_{0}<\Lambda_{0}$, by Theorems 3.3 and 3.6, we obtain (1.1) has two positive solutions $u_{\lambda}$ and $U_{\lambda}$ such that $u_{\lambda} \in \mathcal{N}_{\lambda}^{+}, U_{\lambda} \in \mathcal{N}_{\lambda}^{-}$. Since $\mathcal{N}_{\lambda}^{+} \cap \mathcal{N}_{\lambda}^{-}=\emptyset$, this implies that $u_{\curlywedge}$ and $U_{\curlywedge}$ are distinct. This completes the proof of Theorem 1.1.

\section{References}

[1] L. Caffarelli, R. Kohn, and L. Nirenberg, "First order interpolation inequalities with weights," Compositio Mathematica, vol. 53, no. 3, pp. 259-275, 1984.

[2] T. Horiuchi, "Best constant in weighted Sobolev inequality with weights being powers of distance from the origin," Journal of Inequalities and Applications, vol. 1, no. 3, pp. 275-292, 1997.

[3] G. Talenti, "Best constant in Sobolev inequality," Annali di Matematica Pura ed Applicata, vol. 110, pp. 353-372, 1976.

[4] S. Secchi, D. Smets, and M. Willem, "Remarks on a Hardy-Sobolev inequality," Comptes Rendus Mathématique, vol. 336, no. 10, pp. 811-815, 2003.

[5] D. Kang, "Positive solutions to the weighted critical quasilinear problems," Applied Mathematics and Computation, vol. 213, no. 2, pp. 432-439, 2009.

[6] B. Abdellaoui and I. Peral, "Some results for semilinear elliptic equations with critical potential," Proceedings of the Royal Society of Edinburgh Section A, vol. 132, no. 1, pp. 1-24, 2002.

[7] A. Ambrosetti, H. Brezis, and G. Cerami, "Combined effects of concave and convex nonlinearities in some elliptic problems," Journal of Functional Analysis, vol. 122, no. 2, pp. 519-543, 1994.

[8] D. Cao and P. Han, "Solutions for semilinear elliptic equations with critical exponents and Hardy potential," Journal of Differential Equations, vol. 205, no. 2, pp. 521-537, 2004.

[9] J. Chen, "Multiple positive solutions for a class of nonlinear elliptic equations," Journal of Mathematical Analysis and Applications, vol. 295, no. 2, pp. 341-354, 2004.

[10] A. Ferrero and F. Gazzola, "Existence of solutions for singular critical growth semilinear elliptic equations," Journal of Differential Equations, vol. 177, no. 2, pp. 494-522, 2001. 
[11] P. Han, "Multiple positive solutions for a critical growth problem with Hardy potential," Proceedings of the Edinburgh Mathematical Society Series II, vol. 49, no. 1, pp. 53-69, 2006.

[12] P. Han, "Multiple solutions to singular critical elliptic equations," Israel Journal of Mathematics, vol. 156, pp. 359-380, 2006.

[13] P. Han and Z. Liu, "Solutions for a singular critical growth problem with a weight," Journal of Mathematical Analysis and Applications, vol. 327, no. 2, pp. 1075-1085, 2007.

[14] P. Han, "Many solutions for elliptic equations with critical exponents," Israel Journal of Mathematics, vol. 164, pp. 125-152, 2008.

[15] T.-S. Hsu and H.-L. Lin, "Multiple positive solutions for singular elliptic equations with concaveconvex nonlinearities and sign-changing weights," Boundary Value Problems, vol. 2009, Article ID 584203, 17 pages, 2009.

[16] S. Terracini, "On positive entire solutions to a class of equations with a singular coefficient and critical exponent," Advances in Differential Equations, vol. 1, no. 2, pp. 241-264, 1996.

[17] M. Bouchekif and A. Matallah, "Multiple positive solutions for elliptic equations involving a concave term and critical Sobolev-Hardy exponent," Applied Mathematics Letters, vol. 22, no. 2, pp. 268-275, 2009.

[18] N. Ghoussoub and X. S. Kang, "Hardy-Sobolev critical elliptic equations with boundary singularities," Annales de l'Institut Henri Poincaré, vol. 21, no. 6, pp. 767-793, 2004.

[19] T.-S. Hsu and H.-L. Lin, "Multiple positive solutions for singular elliptic equations with weighted Hardy terms and critical Sobolev-Hardy exponents," Proceedings of the Royal Society of Edinburgh Section A, vol. 140, no. 3, pp. 617-633, 2010.

[20] T.-S. Hsu, "Multiple positive solutions for semilinear elliptic equations involving Hardy terms and critical Sobolev-Hardy exponents," Journal of Mathematical Sciences, vol. 3, no. 2, pp. 243-266, 2009.

[21] D. Kang and S. Peng, "Positive solutions for singular critical elliptic problems," Applied Mathematics Letters, vol. 17, no. 4, pp. 411-416, 2004.

[22] D. Kang and S. Peng, "Solutions for semilinear elliptic problems with critical Sobolev-Hardy exponents and Hardy potential," Applied Mathematics Letters, vol. 18, no. 10, pp. 1094-1100, 2005.

[23] D. Kang and G. Li, "On the elliptic problems involving multi-singular inverse square potentials and multi-critical Sobolev-Hardy exponents," Nonlinear Analysis. Theory, Methods \& Applications, vol. 66, no. 8, pp. 1806-1816, 2007.

[24] Y. Y. Li and C. S. Lin, "A nonlinear elliptic PDE with two Sobolev-Hardy critical exponents," Archive for Rational Mechanics and Analysis, vol. 203, no. 3, pp. 943-968, 2012.

[25] B. Abdellaoui, V. Felli, and I. Peral, "Existence and nonexistence results for quasilinear elliptic equations involving the $p$-Laplacian," Bollettino della Unione Matematica Italiana Sezione B, vol. 9, no. 2, pp. 445-484, 2006.

[26] R. Filippucci, P. Pucci, and F. Robert, “On a $p$-Laplace equation with multiple critical nonlinearities," Journal de Mathématiques Pures et Appliquées, vol. 91, no. 2, pp. 156-177, 2009.

[27] N. Ghoussoub and C. Yuan, "Multiple solutions for quasi-linear PDEs involving the critical Sobolev and Hardy exponents," Transactions of the American Mathematical Society, vol. 352, no. 12, pp. 57035743, 2000.

[28] P. Han, “Quasilinear elliptic problems with critical exponents and Hardy terms," Nonlinear Analysis. Theory, Methods \& Applications, vol. 61, no. 5, pp. 735-758, 2005.

[29] T.-S. Hsu, "Multiplicity results for $p$-Laplacian with critical nonlinearity of concave-convex type and sign-changing weight functions," Abstract and Applied Analysis, vol. 2009, Article ID 652109, 24 pages, 2009.

[30] T.-S. Hsu, "Multiple positive solutions for a quasilinear elliptic problem involving critical SobolevHardy exponents and concave-convex nonlinearities," Nonlinear Analysis. Theory, Methods E Applications, vol. 74, no. 12, pp. 3934-3944, 2011.

[31] D. Kang, "On the quasilinear elliptic problems with critical Sobolev-Hardy exponents and Hardy terms," Nonlinear Analysis. Theory, Methods \& Applications, vol. 68, no. 7, pp. 1973-1985, 2008.

[32] D. Kang, L. Wang, and Q. Wei, “Multiple positive solutions for $p$-Laplace elliptic equations involving concave-convex nonlinearities and a Hardy-type term," Nonlinear Analysis. Theory, Methods $\mathcal{E}$ Applications, vol. 74, no. 2, pp. 626-638, 2011.

[33] P.-L. Lions, "The concentration-compactness principle in the calculus of variations. The limit case- - I," Revista Matemática Iberoamericana, vol. 1, no. 1, pp. 145-201, 1985. 
[34] P.-L. Lions, "The concentration-compactness principle in the calculus of variations. The limit caseII," Revista Matemática Iberoamericana, vol. 1, no. 2, pp. 45-121, 1985.

[35] I. Ekeland, "On the variational principle," Journal of Mathematical Analysis and Applications, vol. 47, pp. 324-353, 1974.

[36] J. F. Yang, "Positive solutions of quasilinear elliptic obstacle problems with critical exponents," Nonlinear Analysis. Theory, Methods E Applications, vol. 25, no. 12, pp. 1283-1306, 1995. 


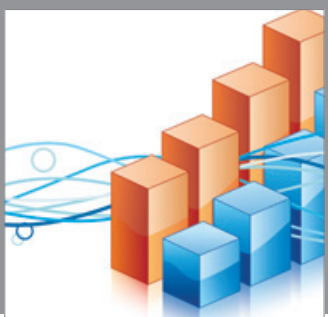

Advances in

Operations Research

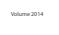

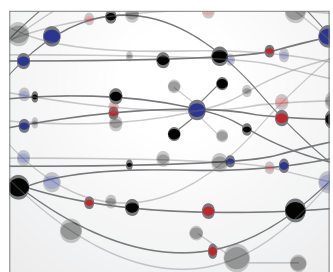

\section{The Scientific} World Journal
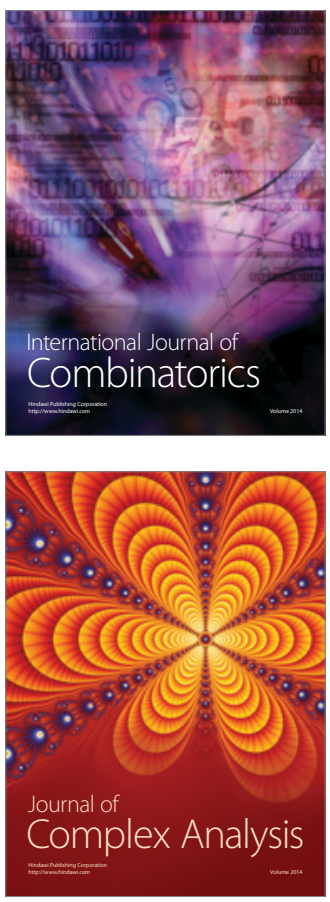

International Journal of

Mathematics and

Mathematical

Sciences
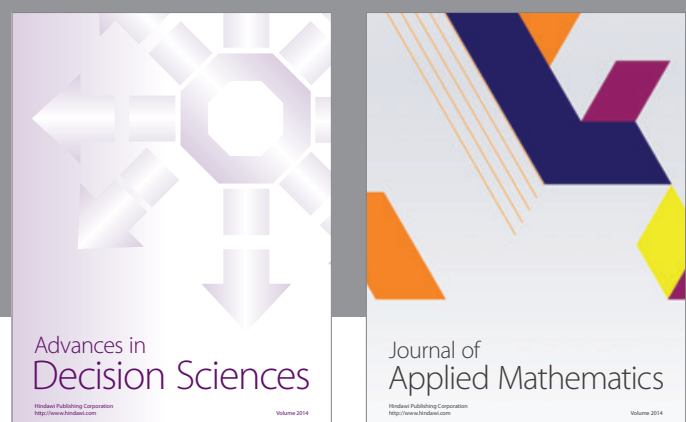

Journal of

Applied Mathematics
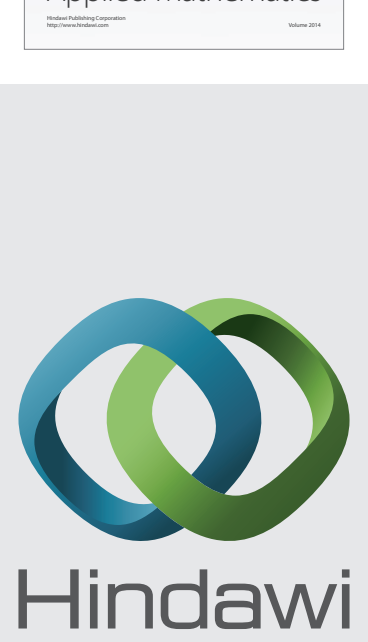

Submit your manuscripts at http://www.hindawi.com
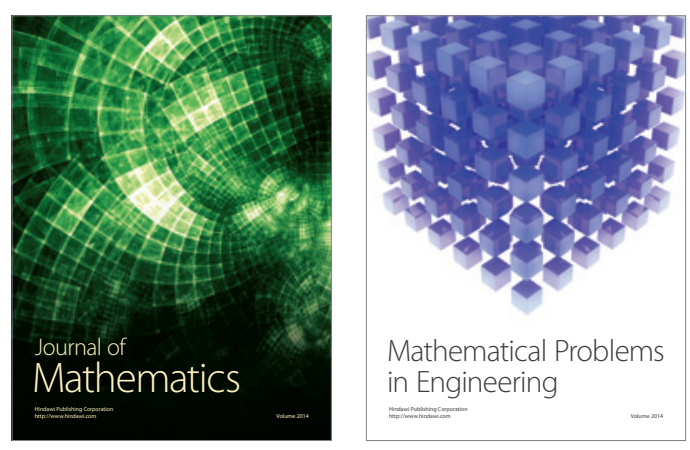

Mathematical Problems in Engineering
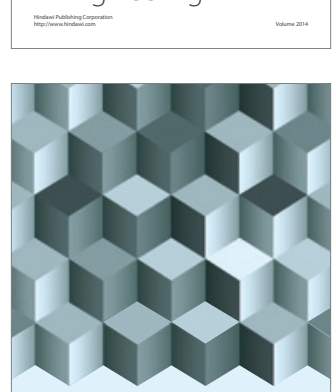

Journal of

Function Spaces
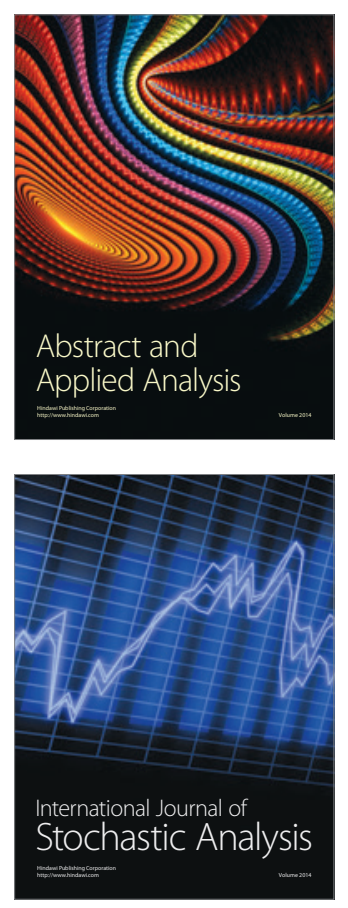

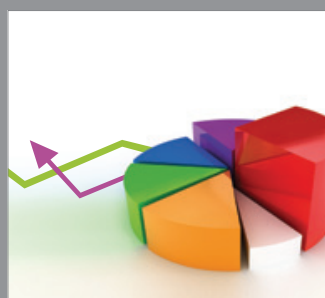

ournal of

Probability and Statistics

Promensencen
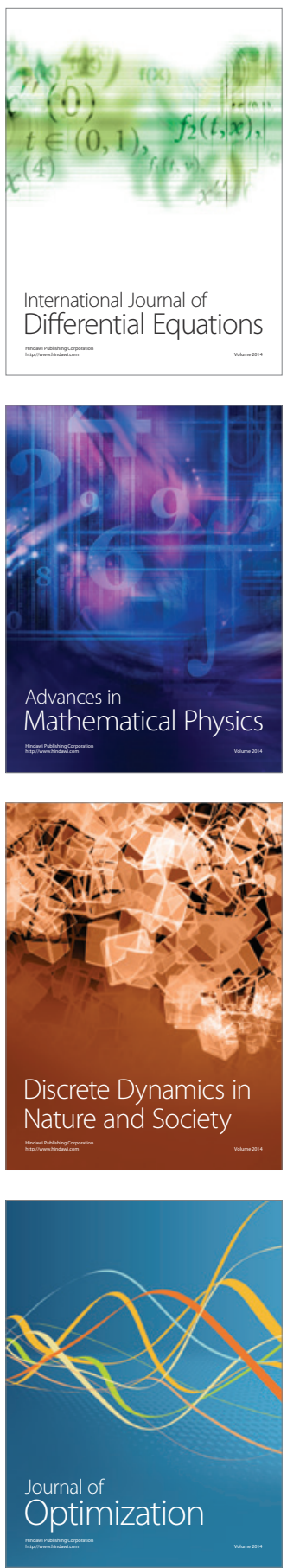